\title{
Analysis, Modeling, and Simulation of Adaptive Control Based on Dynamic Conductance Estimation of Photovoltaic Generator Interfaced Current-Mode Buck Converter
}

\author{
Yakov Malinkovich ${ }^{1}$, Simon Lineykin ${ }^{2}$, Moshe Sitbon ${ }^{1, *}$ \\ ${ }^{I}$ Department of Electrical Engineering and Electronics, Ariel University, \\ Ariel 40700, Israel \\ ${ }^{2}$ Department of Mechanical Engineering and Mechatronics, Ariel University, \\ Ariel 40700, Israel \\ moshesi@ariel.ac.il
}

\begin{abstract}
In this paper, a novel adaptive control method is presented, aimed at robustifying the terminal voltage of the photovoltaic generator, interfaced by the current-modecontrolled buck DC-DC converter load, and based on conductance estimation. The photovoltaic generator, which is integrated into the buck converter and a battery storage unit, is continuously affected by the operating point of the system and environmental variables, thus presenting a nonlinear behavior. Furthermore, the development of small-signal equations reveals a potentially unstable condition when the system is used as a micro-grid with a battery storage unit. This study shows that when the nonlinear behavior of the photovoltaic generator is combined with a typical nominal controller, designed for a single nominal operating point and due to the possibility of an unstable condition, it forces the controller to operate mostly outside the nominal operating point. These conditions result in significantly varied closedloop performance. In contrast, an almost perfect loop gain performance can be achieved when implementing an adaptive controller based on an online conductance estimation method. Applying estimator results and injecting its value in real-time into the inverse-based plant controller results in an adaptive controller. Therefore, the closed-loop performance of the system integrated with an adaptive controller achieves an almost nominal response throughout the operating range.
\end{abstract}

Index Terms-PVG; Adaptive controller; Estimator; Microgrid.

\section{INTRODUCTION}

Renewable energy-based systems, such as photovoltaic generators (PVG) [1], are based on the extraction of the maximum possible energy [2] from a solar source [3] by exploiting a wide range of maximum power point tracking (MPPT) algorithms [4]. However, particularly in the last decade, due to significant progress in microgrid concept development [5], photovoltaic energy generators are now required to operate at a wide range of operating points [6], in addition to the maximum power point (MPP) [7]. Consequently, matching generation and consumption levels

Manuscript received 30 May, 2021; accepted 14 January, 2022. in isolated microgrids [8] are of primary importance [9]. Studies have shown that the properties of the source significantly influence the dynamics of the power converter [10]. One of the variables subject to significant variation due to different operating points is the dynamic resistance of PVG. Hence, the dynamics of the combined PVG-converterload system undergoes substantial changes.

Nowadays, due to significant developments in computing and electronics, there is an increasing requirement for more reliable power electronics systems with high efficiency and consistent responses [11]. Furthermore, renewable energy, which is commonly controlled by power electronics and requires maximum quality and efficiency, shows an advanced improvement and significant advancement in technology, such as in the electric vehicle industry and renewable energy, leading to extensive growth in performance and technology [12]. Consequently, rapid progress in developments calls for enhanced accuracy and performance [13] and robust photovoltaic generation systems [14]. Recent extensive storage capability developments have reduced the size of power electronics devices and increased their efficiency, accelerating the development of microgrids. As a result of this progress, and in contrast to the conventional way of relying only on MPPT control, new requirements for renewable energy sources are now in demand [15]. A storage unit, such as a Lithium-ion battery requires compatibility between power generation [16] and demand [17] when implemented in independent power generation systems [18]. As a result, from the control perspective, the system presents major challenges [19].

Many studies have already established that the operating voltage of the photovoltaic generator is determined by the variables of environmental conditions and directly determines the output power [20]. Consequently, the internal resistance of the PVG is dramatically altered due to its direct dependence on environmental conditions and operating points [21]. Therefore, implementing a nominal linear controller in a PVG-converter-load system, which is 
designed with a single nominal point, will result in significantly different closed-loop performance [22]. By contrast, when a controller-based disturbance observer is implanted, the response will be constant, but nominal. That is, the response rate and shape will be according to the worst-case scenario, which is the least optimal to maintain stability throughout the operating range [23]. Therefore, from a control perspective, the use of the Buck converter forces the system to choose the lower permitted PVG voltage [24] and not the common operating point, which is around MPP. To track the changes in real-time of the dependency of the variable PVG-converter-load on the operating point, an implementation of an adaptive control method is required to achieve consistent and nominal closed-loop performance [25] throughout the whole operation range [26].

In this paper, adaptive control is demonstrated for online dynamic conductance estimation based on the PVG-buck power stage-load. Additionally, we take into account the stability problems that arise from the use of a buck converter. Also, our study responds to the need to reshape the plant according to small-signal analysis results for simple implementation. In control design, the goal is to achieve perfect loop gain by using the inverse plant as the controller base. However, in this case, due to a significant change of the dynamic conductance along with the operation range and the possibility of a right pole formation, the inverse plant cannot be directly used to construct this type of controller. Therefore, an online adaptive method should be implemented to handle the uneven response and the right pole. The adaptive controller base is the estimator, which evaluates dynamic conductance in real-time and injects the estimated value into the controller. Hence, by adapting the controller variable values in real-time to the operating point, the voltage control loop can interface with the actual plant and regulate it with an inverse plant-based controller. Additionally, to solve the influence and appearance of the right pole and ensure stability in any case, a small constant, based on static conductance of the minimum operating point and exerting a minor influence on the overall response, is added to the controller.

In this work, the dynamic conductance estimator is implemented using the method presented in [4], on a modified and reduced plant due to system complicity and right pole feasibility. Consequently, a fast and accurate estimation of the main component, internal resistance, and modification of the controller value in real-time actually creates an inverse nominal plant almost identical to the actual inverse plant, although it depends on the operating point and environmental conditions. Thus, it maintains an almost constant closed-loop performance throughout the operating range.

The rest of the paper is organized as follows. The system description is given in Section II. The problem formulation is given in Section III. The estimator method is given in Section IV. Adaptive control principles, implementation, and verification are given in Section $\mathrm{V}$. The paper is concluded in Section VI.

\section{SYSTEM DESCRIPTION}

PVG is typically interfaced by a buck-boost or boost converter due to stability issues and simple plant components. On the contrary, this paper illustrates the applicability of a buck power stage (BuPS). A PVG-BuPSLoad control structure is usually assembled from two loops: an external voltage loop and an internal current loop (with which it is commonly integrated), mainly to construct overcurrent protection, reduce system order, and increase robustness. Such a cascaded control structure is shown in Fig. 1. To analyze the PVG-BuPS-Load system, a voltage loop small-signal analysis is formed. Due to the slow variation of the load properties [27], the dynamic is mainly dependent on the PVG and the converter. In addition, the limitation of interfacing a buck converter in a microgrid, which is built to charge a battery storage unit, should be considered [28]. For safety reasons, the battery's upper limit voltage of operation should be taken into account since it determines the PVG minimum voltage of operation. That is, the internal voltage of the buck converter must be higher than the output voltage, thus practically determining the minimum possible PVG operating voltage.

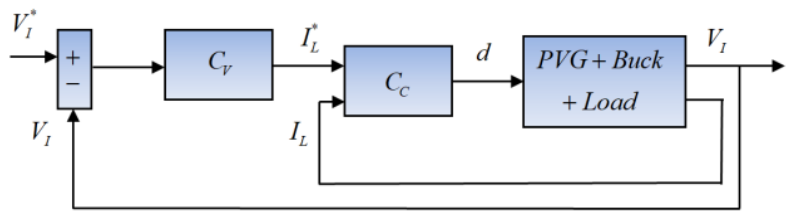

Fig. 1. System control structure.

\section{PROBLEM FormULATION}

The system under consideration is shown in Fig. 2, when the PVG is connected to the internal BuPS capacitor $C_{I}$. In general, the PVG is terminated by a DC-AC inverter connected to the grid, whereas in this paper a DC-DC buck converter is used as part of an autonomous system, being connected to a battery storage unit when the overall system is used as a portable Lithium-ion battery charger. Taking advantage of this kind of system to charge a battery storage unit means that the load is of a voltage source type and can be represented by a Thevenin equivalent circuit with slowly varying parameters [27]

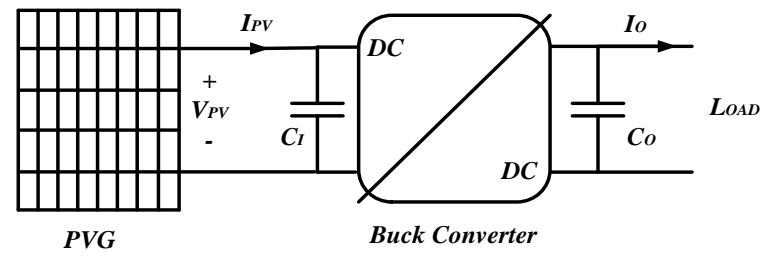

Fig. 2. PVG interfaced with the Buck converter.

To represent the PVG in electrical form, an equivalent circuit diagram is offered in Fig. 3(a). The components that illustrate the PVG equivalent model are $I_{P V}$ as the photocurrent source, with $R_{S H}$ as the shunt resistance, and $C_{P V}$ as the capacitance, respectively. $R_{S}$ is the series resistance, and $I_{D}$ illustrates the recombination loss current [29]. 


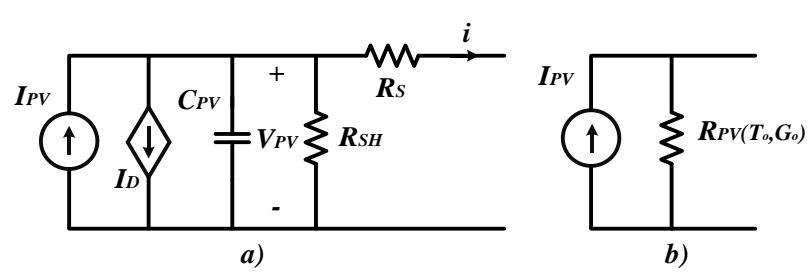

Fig. 3. PVG equivalent circuit: (a) Detailed; (b) Norton.

Although Fig. 3(a) shows a typical and straightforward PVG electrical representation, where $R_{S H}$ and $R_{S}$ are commonly considered as constants, in reality, $R_{S H}$ is irradiance-dependent and, additionally, a component that cannot be measured directly and has a significant influence is the dynamic resistance, $R_{D} . R_{D}$ is produced as a result of recombination losses, which are current temperature dependent and must be considered. The recombination loss current can be presented in a general form as

$$
I_{D}=\sum_{k} I_{0 k}\left(\exp \left\{\frac{V_{P V G}-R_{S} I_{P V G}}{\alpha_{k} V_{T}}\right\}-1\right),
$$

with $I_{o k}, a_{k}$, and $V_{T}$ denoting the reverse saturation current, the ideality factor of the $k_{t h}$ diode, and the thermal voltage, respectively.

Consequently, the equivalent Norton resistance $R_{P V}$ will be shaped by the resistance and recombination current. Hence, it can be represented as the dynamic resistance (irradiance $G_{0}$ and temperature $T_{0}$ dependent) Norton equivalent

$$
R_{P V}\left(G_{0}, T_{0}\right)=R_{S}+R_{S H} \| R_{D}
$$

To calculate the Norton equivalent, the capacitor $C_{P V}$ can be neglected, mainly due to its small scale and due to the elimination by the internal capacitor of the converter. Thus, after considering all the given components, a PVG dynamic Norton equivalent circuit, environmental variables, and operating point dependence can be applied (see Fig. 3(b)).

The second part of the integrated PVG system is the DCDC buck converter, where the electric model (a) and the average model (b) are presented in Fig. 4. The switching cycle of the electric model is illustrated as the current and voltage sources, dependent on the duty cycle, as shown in the average model.

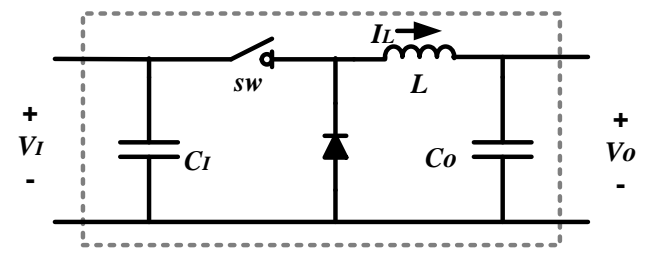

(a)

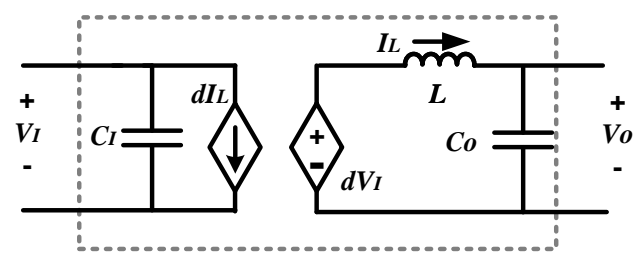

(b)

Fig. 4. Buck converter, (a) electric (up) and (b) average (down) models.
Complementary to the integrated system is the load/storage unit, which commonly uses a Lithium-ion battery in microgrid concepts and portable battery charger PVG-based systems. Therefore, it is exploited in this paper due to its extensive use and characteristic features. The electric battery model is shown in Fig. 5, and the equivalent electrical circuit is built from the internal resistance $R_{B}$ and the voltage source $V_{B}$ with a slow variation. The internal resistance $R_{B}$ possesses a small-scale value, and so the voltage drop on $R_{B}$ is directly influenced by the overall battery voltage when charging or discharging, respectively, to the current.

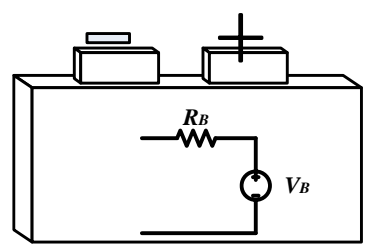

Fig. 5. Battery Thevenin equivalent.

Integrating all the components of the electric models allows one to construct a PVG-BuPS-Load average model system; see Fig. 6, where $L$ and $d$ represent converter inductor and duty cycle, respectively, while $C_{I}$ and $C_{o}$ are internal and external capacitors, respectively, $V_{I}$ and $V_{o}$ are converter internal and external voltages, and $I_{L}$ is inductor current, $I_{P V}$ and $R_{P V}$ are the PVG Norton parameters, while $R_{B}$ and $V_{B}$ denote load Thevenin parameters.

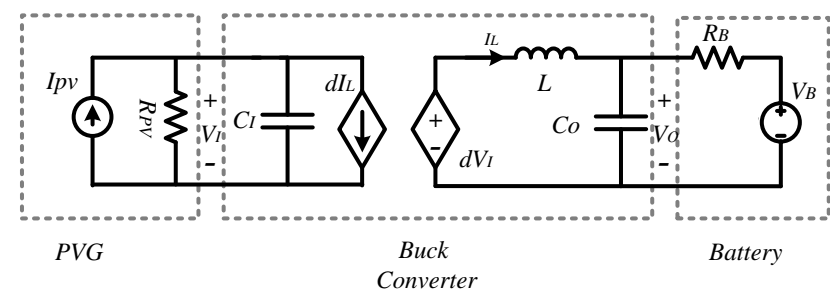

Fig. 6. An average model of the combined PVG-BuPS-Load system.

Analyzing a small-signal average model, assuming continuous conduction mode according to the formula developed in [24], yields the following set of equations:

$$
\left\{\begin{array}{l}
C_{I} \dot{\tilde{v}}_{I}=-R_{P V}^{-1} \tilde{v}_{I}-I_{L} \tilde{d}-D \tilde{i}_{L}, \\
L \dot{\dot{\tilde{i}}_{L}}=D \tilde{v}_{I}+V_{I} d-\tilde{v}_{O}, \\
C_{O} \dot{\tilde{v}}_{O}=\tilde{i}_{L}-R_{B A T}^{-1} \tilde{v}_{O} .
\end{array}\right.
$$

This set can be linearized around an operating point given by:

$$
\left\{\begin{array}{l}
\frac{P_{P V}}{V_{I}}=I_{P V}-V_{I} R_{P V}^{-1}=D I_{L}, \\
D V_{I}=V_{O}, \\
\frac{P_{P V}}{V_{O}}=I_{L}=\left(V_{O}-V_{B A T}\right) R_{B A T}^{-1} .
\end{array}\right.
$$

Keeping in mind the order of the loops of the control structure and the need to meet bandwidth requirements, when $C_{V}$ and $C_{C}$ are the voltage control and current control, respectively, as shown in Fig. 1, and if the current loop is 
appropriately closed and its bandwidth is much higher than the voltage loop bandwidth, perfect current tracking may be assumed

$$
\tilde{i}_{L}(t) \cong \tilde{i}_{L}^{*}(t)
$$

Substitution of (5) into (3), using the Laplace transform and rearranging the results, gives

$$
\frac{\tilde{v}_{i}}{\tilde{i}_{L}^{*}}(s)=-\frac{D+\frac{I_{L}}{V_{I}} L s}{C_{i} s+R_{P V}^{-1}-R_{s}^{-1}},
$$

with

$$
R_{s}^{-1}=\frac{I_{L}}{V_{I}} D .
$$

Close examination of the receiving transfer function (6) enables prominently to see the minus sign of the denominator. Hence, the influence of $R_{P V}$ and $R_{S}$ values on the transfer function stability is of prime importance. Also, as mentioned, their values undergo dramatic changes due to ongoing changes of operating point and environmental conditions. Consequently, due to the variables that possess the stability of the dominator, their sum values can lead to an unstable pole when the operating point is on the lower voltage side of the PV curve. Hence, a careful design is needed if the load is a Lithium-ion battery, in which case, the upper and lower limits of the safety battery voltage should be considered. These limitations require ongoing adjustment in the operation of the PV voltage, which practically yields a variety of responses, depending on the position of the operating point. Hence, the system response does not possess a nominal response and practically shifts from overdamped to underdamped to the point of instability. A reshaped transfer function, combined with a real-time adaptive controller, is presented in this paper to overcome the controlling complexity through the entire range of operation.

First, it should be acknowledged that the converter output voltage parameter is restricted between two boundaries, which are determined by the load, which in our case is a Lithium-ion battery. Due to physics and safety reasons, the upper and lower voltage limits must be kept, a limitation that directly influences the PVG output voltage. Accordingly, this effect directly influences the BuPS input voltage. That is, the fact that the PVG voltage dynamic is mainly influenced by environmental conditions and the operating point over the I-V curve must be considered. Traditionally, a large capacitor is used to eliminate these influences when using a DC/DC converter or when the maximum power was the controlling target. However, considerable research has gone into reducing the capacitor size and consequently reducing the system size. Consequently, there has been a significant improvement in the overall life of the system, mainly due to the use of ceramic capacitors. Therefore, when using a PVG and a Lithium-ion battery as a sink, internal dynamics produce a major effect on the shape of the PVG response, despite the use of a large capacitor [28].

Analyzing a normalized standard, the random PVG I-V curve for haphazard temperature $T_{0}$ and irradiance $G_{0}$ is shown in Fig. 7.

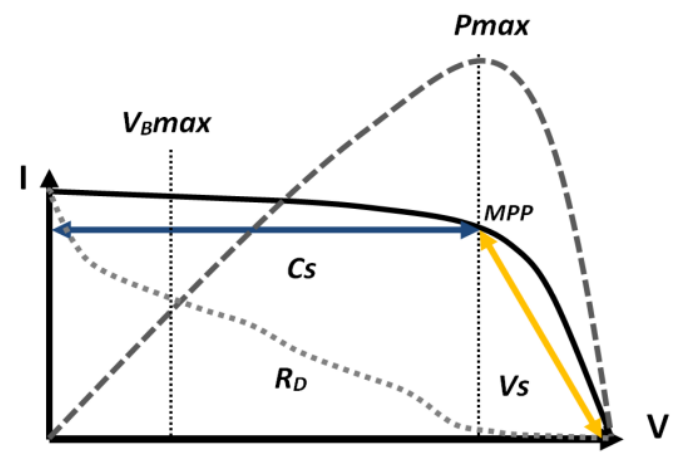

Fig. 7. PVG I-V curve.

It is possible to split the I-V curve into two parts due to different behavior before and after the MPP point. As is clearly shown, the PVG conducts itself as a current source $\left(C_{s}\right)$ (blue arrow) at voltages before the MPP voltage and as a voltage source $\left(V_{s}\right)$ (yellow arrow) at voltages after it. The significant difference in performance directly influences the internal resistance values; that is, the current source possesses a high dynamic resistance compared to the voltage source area.

When studying the receiving transfer function (6) from a stability perspective, an unstable pole can occur at

$$
\left(R_{P V}^{-1}-R_{s}^{-1}\right)<0 .
$$

In this paper, an autonomous charging system is used. Therefore, the $R_{P V}$ will fluctuate considerably due to the dependency on the operating point. Consequently, the outcome of $\left(R_{P V}^{-1}-R_{s}^{-1}\right)$ will determine the stability and response of the system. Hence, two major drawbacks need to be solved for creating a perfect loop gain controller of the form

$$
C(s)=-\frac{\omega_{c}}{s}\left(P^{-1}\right)
$$

These are to maintain stability and to estimate dynamic resistance throughout the operating range. Therefore, careful design and adaptive mechanisms should be used and implemented. When considering the operating range limits of an autonomous system, a storage unit such as a Lithiumion battery significantly influences the PVG operating point due to safety limits. Consequently, one needs to consider the buck converter properties, whereas the upper voltage safety boundary of the storage unit $\left(V_{B \max }\right)$ actually states the minimum PVG voltage of operation, see Fig. 7. Therefore, the adaptive controller should be designed to hold stability all over the operating range and reflect the designed response no matter what the conditions are. Due to the significantly complex plant of the PVG-BuPS-Load and the requirement to implement it in an inverse way into the perfect loop gain controller, a few system plant 
manipulations are needed. By doing so, a reduced and simpler plant is achieved. However, the reduced plant should perform identically to the original plant in the bandwidth of interest. The transfer function can be written as follows

$$
P(s)=\frac{\tilde{v}_{i}}{\tilde{i}_{L}^{*}}=-\frac{D\left(1+\frac{L I_{L}}{V_{I} D} s\right)}{\left(R_{P V}^{-1}-R_{s}^{-1}\right)\left(1+\frac{C_{i}}{R_{P V}^{-1}-R_{s}^{-1}} s\right)},
$$

where the gain can be presented as

$$
G=\frac{D}{\left(R_{P V}^{-1}-R_{s}^{-1}\right)}
$$

The part that can be neglected is as follows: the bandwidth of interest is at lower frequencies. Hence, we can assume that the rest of the numerator approaches one (12)

$$
1+\frac{L I_{L}}{V_{I} D} s \rightarrow \approx 1
$$

Furthermore, for more simplicity, $\omega_{p v}$ is defined as

$$
\omega_{p v}=\frac{R_{P V}^{-1}-R_{s}^{-1}}{C_{i}}=\frac{Y_{P V}-Y_{s}}{C_{i}}
$$

eventually resulting in the reduced plant

$$
P_{\mathrm{r}}(s)=\frac{\tilde{v}_{i}}{\tilde{i}_{L}^{*}} \approx \frac{-G}{\left(\omega_{P V}^{-1} s+1\right)}
$$

Placing the variables allows one to reduce the transfer function to

$$
P_{\mathrm{r}}(s)=-\frac{D}{\left(Y_{P V}-Y_{s}\right)+C_{i} s} .
$$

The Bode response is used to validate the reduced transfer function $P_{r}$ (15) due to neglected parts from the primary transfer function, such as $V_{O}$, load $\left(V_{B A T}, R_{B A T}\right)$, and neglected zero Bode response is used. Applying values from Tables I, II, and III, which present real PV and Battery values, illustrates the performance from the Bode curve (Fig. 8) perspective, whereas the blue line illustrates the Bode response of the primary transfer function including all the acquired plant parts of small-signal analysis.

TABLE I. BUCK CONVERTER SPECIFICATIONS.

\begin{tabular}{|c|c|}
\hline Nominal power & $120[\mathrm{~W}]$ \\
\hline Input capacitance $\mathbf{C}_{\boldsymbol{I}}$ & $470[\mu \mathrm{F}]$ \\
\hline Inductance $\mathbf{L}$ & $100[\mu \mathrm{H}]$ \\
\hline
\end{tabular}

TABLE II. PVG SPECIFICATIONS AT STC.

\begin{tabular}{|c|c|}
\hline Nominal power & $120[\mathrm{~W}]$ \\
\hline MPP voltage & $23[\mathrm{~V}]$ \\
\hline MPP current & $5.1[\mathrm{~A}]$ \\
\hline Open circuit voltage & $29[\mathrm{~V}]$ \\
\hline Short circuit current & $5.9[\mathrm{~A}]$ \\
\hline
\end{tabular}

TABLE III. BATTERY SPECIFICATIONS

\begin{tabular}{|c|c|}
\hline Capacity & $15.6[\mathrm{~A} / \mathrm{h}]$ \\
\hline Maximum voltage & $16.8[\mathrm{~V}]$ \\
\hline Minimum voltage & $14.4[\mathrm{~V}]$ \\
\hline
\end{tabular}

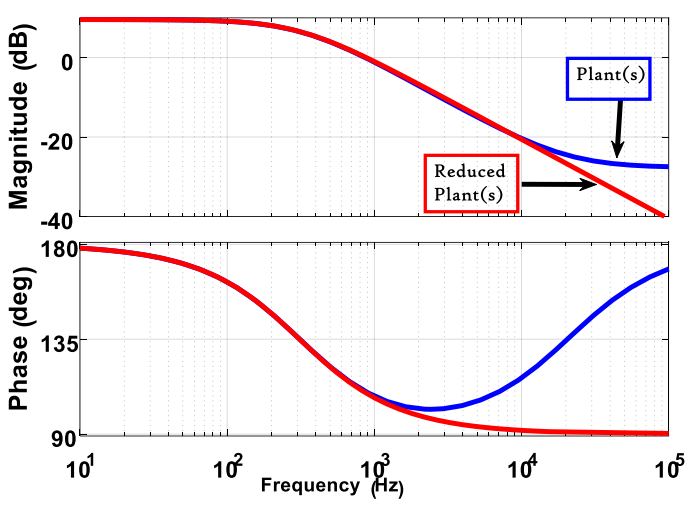

Fig. 8. Bode response of complete $(P(s))$ and simplified $\left(P_{r}(s)\right)$ plants.

On the contrary, the red line represents the incomplete transfer function, which neglects some of the primary transfer function components. Careful examination of the Bode diagram reveals that the lines exhibiting the same responses are correlated at the operating zone from a frequency perspective. Consequently, the overall system can respond as designed when applying the unfull transfer function (15) at the bandwidth of interest.

According to the reduced transfer function $P_{r}(s)(15)$ and the form of the chosen controller $C(s)(9)$, implementing the inverse transfer function $P_{-r}^{-1}(s)$ in the controller will receive the following form

$$
C(s)=\frac{\omega_{c}}{s} P_{r}^{-1}=-\frac{\omega_{c}}{s} \frac{\left(\hat{Y}_{P V}-Y_{s}\right)+C_{I} s}{D} .
$$

Consequently, the overall open-loop of the reduced transfer function with controller, for any given operating point and environmental condition, and if the estimation ( $\hat{Y}_{P V}$ estimated conductance) is correct, is as follows

$$
O L_{\mathrm{P}_{r}+C}(s)=\frac{\omega_{c}\left(\left(\hat{Y}_{P V}-Y_{s}\right)+C_{I} s\right) D}{s D\left(\left(Y_{P V}-Y_{s}\right)+C_{I} s\right)}=\frac{\omega_{c}}{s} .
$$

Hence, the closed-loop can perform as a first-order overdamped system, and therefore, it can even perform as a perfect loop gain

$$
C L_{\mathrm{P}_{r}+C}(s)=\frac{\omega_{c}}{s+\omega_{c}}=\frac{1}{\frac{1}{\omega_{c}} s+1}=\frac{1}{\tau s+1}, \quad \tau=\frac{1}{\omega_{c}} .
$$

Even so (i.e., meeting the goal of perfect loop gain response), a few significant drawbacks should be resolved first. Dynamic conductance is an environmental condition and depends on the operating point variable. However, $Y_{s}$ is directly determined by current, voltage, and duty cycle (7). Practically, these variables are easy to measure and use. In contrast, dynamic conductance $Y_{P V}$ is more complex and cannot be directly measured, and, in addition, it depends on 
the environmental condition and operating point, as shown in Fig. 7, and can be represented as $R_{D}^{-1}$ (dynamic resistance). Hence, some kind of estimator mechanism should be implemented for real-time estimation of dynamic resistance, and further, it should be injected into the inverse controller as dynamic conductance. Consequently, after implementing the estimator and basic measurements, the adaptive controller should be as follows

$$
C_{\text {Adaptive }}(s)=-\frac{\omega_{c}}{s} \frac{\left(\hat{Y}_{P V}-\vec{Y}_{s}\right)+\left(C_{i} s\right)}{\vec{d}},
$$

where $\hat{Y}_{P V}$ is the estimating dynamic conductance and $\vec{Y}_{s}$ and $\vec{d}$ are variables extracted from the measurement of voltage, current, and duty cycle.

The second drawback is the possibility that an unstable pole can appear at a lower operating voltage, as expressed in (8). To solve this problem at any operating point, a small constant value will be added to the system that depends on the characteristics of the PVG to ensure stability throughout the operating range without a significant influence on the response of the system. This method will be explained later.

\section{ESTIMATOR METHOD}

To evaluate dynamic conductance, as done in [4], we need to force the system to oscillate at a high frequency and very low amplitude. Hence, a fixed-amplitude artificial oscillation signal, such as

$$
i_{d}(t)=I_{D} \sin \omega_{0} t
$$

is intentionally injected into the voltage controller output, thus adding a small oscillating sinus wave to the current controller reference. It should be noted that $\omega_{O}$ should be set as high as possible to increase the estimation bandwidth. However, it should be about ten times lower than the current loop bandwidth to allow precise current tracking; that is, it will allow us to assume that $i_{L} \cong i_{L}^{*}+i_{d}$ from the point of view of the voltage loop. Note that, since the input voltage loop bandwidth must be at least five times lower than the dynamic resistance estimation period, increasing the estimation bandwidth allows increasing the input voltage loop bandwidth.

The overall base system combined with an estimator is illustrated in Fig. 9, where the measured PVG voltage and inductor current are transferred to the estimator. In addition, a fixed-amplitude artificial oscillation signal is injected into the estimator and the output of the voltage controller.

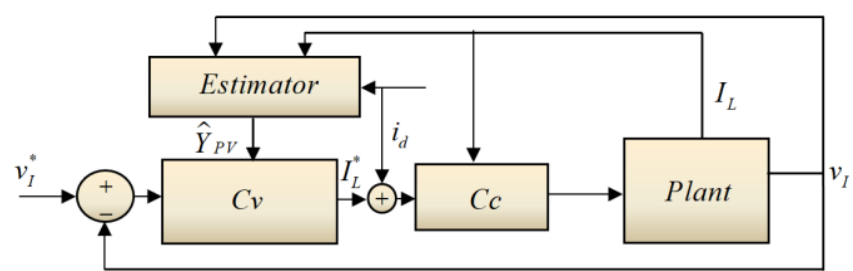

Fig. 9. Based estimator system.

The artificial oscillation $i_{d}$-to-input voltage transfer function is given by

$$
\frac{\tilde{v}_{i}}{\tilde{i}_{d}}(s)=-\frac{D s}{\left(R_{P V}^{-1}-R_{s}^{-1}+C_{I} s\right)\left(s+\omega_{c}\right)},
$$

i.e., for $\omega_{O} " \omega_{c}$ its magnitude at $s=j \omega_{O}$ is

$$
\left|Z_{d}\right| \square\left|\frac{\tilde{v}_{i}}{\tilde{i}_{d}}\left(s=j \omega_{0}\right)\right| \cong \frac{D}{\sqrt{\left(R_{P V}-R_{s}\right)^{-2}+\left(\omega_{0} C_{I}\right)^{2}}},
$$

and may be calculated as

$$
\left|Z_{d}\right|=\frac{R M S\left(B P F_{\omega_{0}}\left\{v_{i}\right\}\right)}{R M S\left(B P F_{\omega_{0}}\left\{i_{d}\right\}\right)}
$$

Consequently, the dynamic resistance of PVG may be estimated as

$$
\hat{R}_{P V}^{-1}=\sqrt{\frac{D^{2}}{\left|Z_{d}\right|^{2}}-\left(\omega_{0} C_{I}\right)^{2}}+Y_{s} .
$$

\section{VERIFICATION}

\section{A. Comparing Method Responses by Bode Diagram}

To assess the system response to different $V_{P V}$ voltages, a loop gain Bode's diagram is used. The typical controller behavior combined with a nominal plant (blue) or with a reduced plant (red) is shown in Fig. 10.

It is clearly shown that the lines are correlated at the frequency of interest at open-loop.

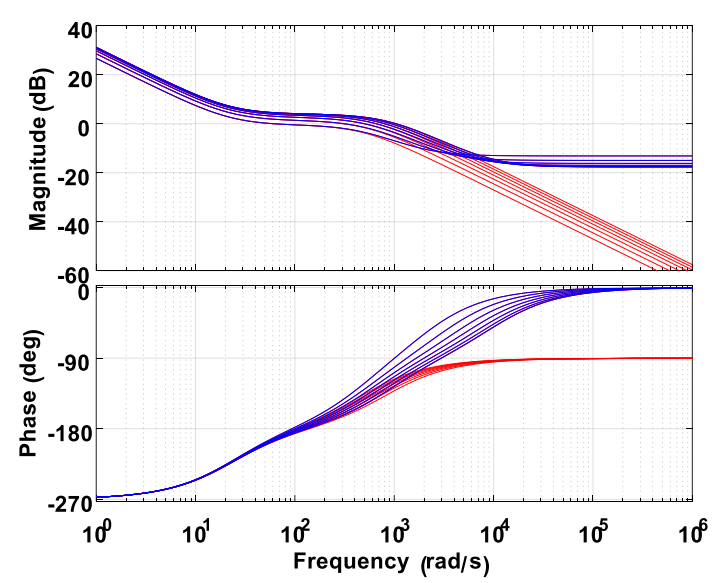

Fig. 10. Bode response of the nominal controller combined with a nominal plant (blue) or with a reduced plant (red)

The results of combining the typical controller and reduced plant $C_{N}(s) P_{r}(s)$ into an ideal adaptive control structure $C_{\text {ideal-adaptive }}(s) P_{r}(s)$ are presented in Fig. 11, with $\omega_{C}=50 \pi \mathrm{rad} \times \mathrm{s}^{-1}, C_{I}^{N}=470 \mu \mathrm{F}, \quad$ and $Y_{P V}^{N}=0$ as the worst-case operating point. Figure 11, representing the Bode response for the open-loop reduced plant (blue lines), is compared to the ideal adaptive control, which leads to a perfect loop gain (green lines). Comparing the response of the loop gains for different operating voltages indicates that the nominal controller has a significantly wide cross-over 
frequency. This will hold only on the assumption that, using a real-time estimator, a linked response of a perfect loop gain can be achieved. Therefore, the cross-over frequency is comparatively narrow, and therefore the system response should be almost identical regardless of the operating point.

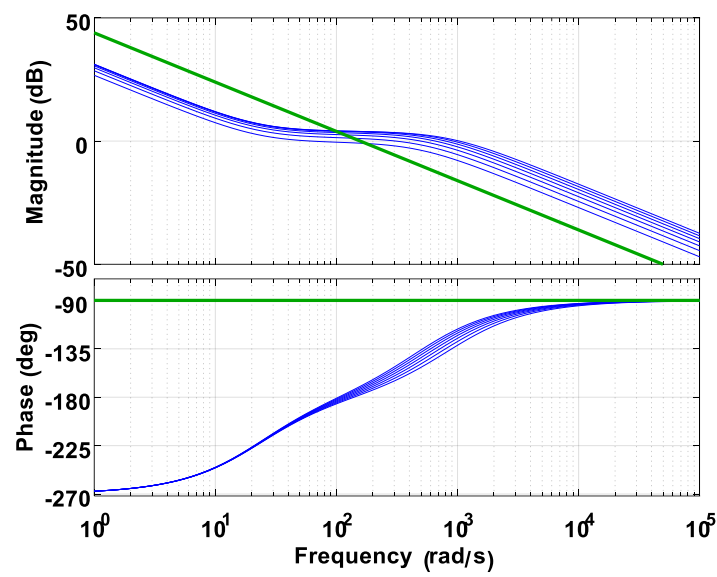

Fig. 11. Bode response for open-loop reduced plant and controller (blue lines) compared to reduced plant and ideal adaptive controller (green lines).

\section{B. Method Performance}

To verify the adaptive capability of the system and to achieve the goal of unified nominal performance throughout the operating range of the PV I-V curve, Tables I, II, and III are used. These tables demonstrate the values and performance of an actual converter and solar panel at 25 Celsius and $1000 \mathrm{~W} / \mathrm{m}^{2}$, which were used to assemble a mini-scale portable Lithium-ion battery charger in practice.

Implementing all system components and combining the estimator yields the overall adaptive control for the integrated PVG-BuPS-Load system, illustrated in Fig. 12.

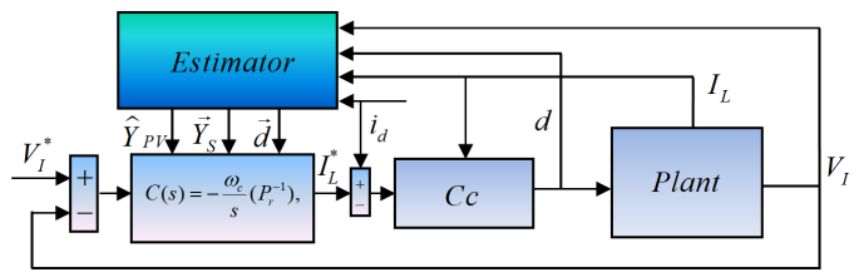

Fig. 12. Overall estimation method.

To demonstrate the variation in closed-loop response, the typical proportional-integral (PI) controller and reduced plant $P_{r}(s)$ are compared with the adaptive controller. As presented in Fig. 12, the method is implemented in the most representative way. Additionally, the constant that is implemented inside the estimator to prevent the right pole is built identically as $Y_{S}(7)$ : that is, using values of voltage, current, and duty cycle with one significant difference. In contrast, the duty cycle value is based on real-time measuring, where voltage and current are constant and selected according to the average value of the minimum $V_{P V}$ operation. Consequently, its influence is minor at higher $V_{P V}$ voltage due to the small value of the duty cycle, and is highest when working at low $V_{P V}$ voltage. That is, the constant possessing new value according to the operating point can counteract the possibility of a right pole while having only a minor influence on the system response. Therefore, for demonstration purposes, different voltage step values are chosen, from the effective operation voltage of the open circuit to the almost lower boundary allowed for the operation of the PV I-V curve (see Fig. 13).

Evaluating the results, we see a significant difference between a reference voltage (green line), which is a change at adequate and sharp steps compared to the over-all system closed-loop response (blue line), which varies considerably when using a typical controller. In addition, the response shape is determined directly according to the voltage operating point, the response shape changes from underdamped to overdamped. On the other hand, the adaptive controller response is almost constant and reflects the perfect loop gain operation. The slight difference in response along the operating point is due to adding the small constant to the estimator to avoid the unstable pole in any case.

For a more profound look, a zoom-in is made in Fig. 14. The zoom-in validates the almost perfect loop gain response in contrast to the nominal response. It is important to note that the nominal response of the typical controller is at the lower limit of operation and is at approximately 17 [V] due to battery characteristics. Even if stability is achieved in the whole range of operations, the considerable fluctuation in response requires implementing adaptive control to ensure a constant response. Furthermore, the adaptive control method has allowed choosing the cross-over frequency, i.e., the system response, without being dependent on the typical controller. For example, the disturbance observer [23] depends on the typical controller, since the proportional constant $\left(k_{p}\right)$ of the typical controller is restricted to the lower level due to possessing the right pole. Consequently, improving the response with constant controller magnification will directly influence the allowed operation bandwidth.

The first test results presented so far were obtained using standardized environmental conditions. In the rest of this section, the system response will be evaluated for different irradiance and temperature. Figure 15 represents the test response of a typical controller for different levels of irradiance (from 1000 to 400 descending steps of $200 \mathrm{~W} / \mathrm{m}^{2}$ ), at a temperature of 25 degrees Celsius.

Figure 15 clearly shows that the results characterize the behavior of the typical controller, which varies considerably along with the operating points, which means that there are no advantages to a typical controller at different irradiances. However, testing the integrated adaptive control reveals its ability to disable the actual plant component variation in real-time, even though the components experiencing significant change. Eventually, it creates an almost perfect loop gain response, as shown in Fig. 16, where the green line represents voltage reference steps, and the rest of the colors represent the response for different irradiances. The small difference in response between the steps is due to the addition of a small constant value to ensure stability for the entire operating range, even though the influence is limited and narrow, and the response shape matches the design. Clear results can be seen in Fig. 17, where a zoom-in is made to evaluate the adaptive response. The almost exponential descent of the curve as an overdamped step response is thus well established. 
The following test is intended to demonstrate the performance of the system at a different temperature (from 20 to 50 ascending steps of $10^{\circ} \mathrm{C}$ ) and the same descending $V_{P V}$ steps are used. Figure 18 shows the response of the typical controller system at different temperatures. It is important to note that temperature is a significant variable that influences the PVG voltage of operation and, as a result, directly impacts the output power, open-circuit voltage, etc. Hence, when analyzing the typical controller response, the transition between underdamped and overdamped is clearly shown in the transition from 23 to 21 volts. When the temperature increases to $40{ }^{\circ} \mathrm{C}$, it directly influences the response.

A thorough examination of the adaptive response to different temperature levels is presented in Fig. 19. This reveals an almost perfect loop gain response independent of the temperature value. In addition, the system responses to

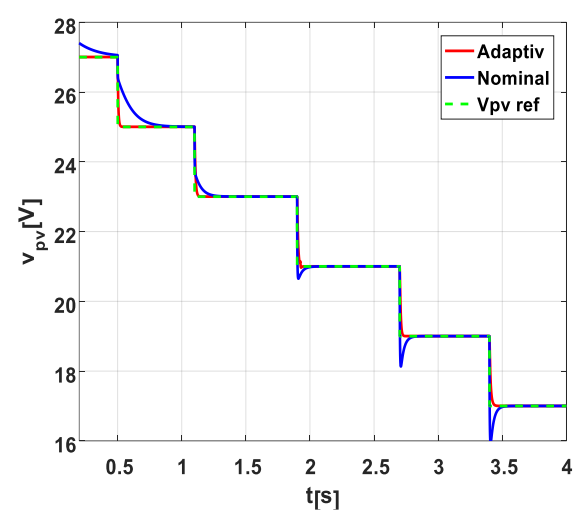

Fig. 13. Step response of a typical PI controller vs. adaptive controller.

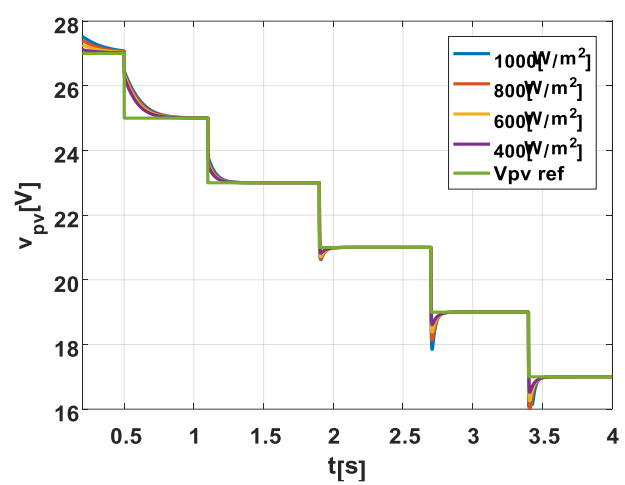

Fig. 15. Typical controller response for different irradiances.

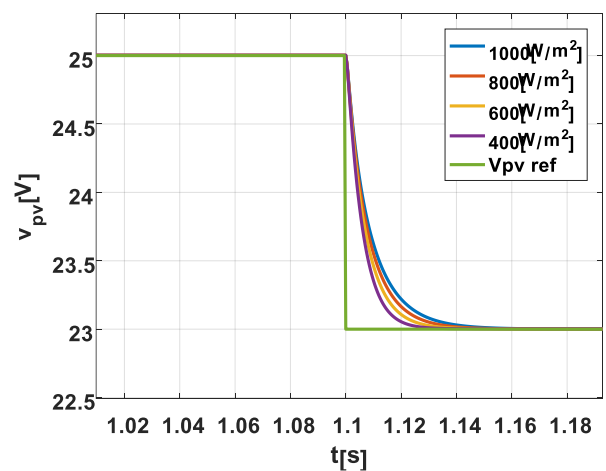

Fig. 17. Zoom-in for the step response of an adaptive controller for different irradiance. changes in irradiance and temperature are almost identical. Consequently, so is establishing the quality of the estimation method, i.e., creating a perfect loop gain under all conditions.

It should be noted that the extensive variation response of the typical controller, not only at a different operating voltage $V_{P V}$, but also at the same operating voltage, directly influences the response due to a significant change of the dynamic conductance value at the same voltage of operation. This effect is due to the different irradiance or temperature and the presence of dynamic conductance in the system transfer function denominator (15). In contrast, as shown in the adaptive zoom-in in Fig. 20, the ability to estimate dynamic conductance in real-time cancels the influence of dynamic conductance, resulting in an almost unified response regardless of the voltage of operation, temperature, or irradiance.

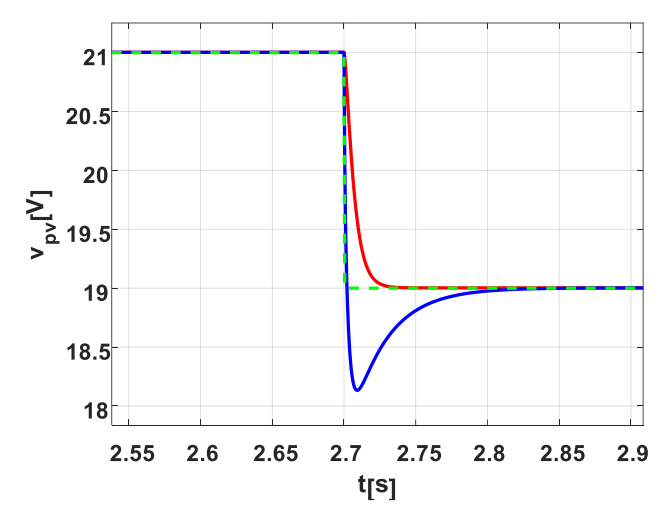

Fig. 14. Zoom-in at step response of a typical controller vs. adaptive controller.

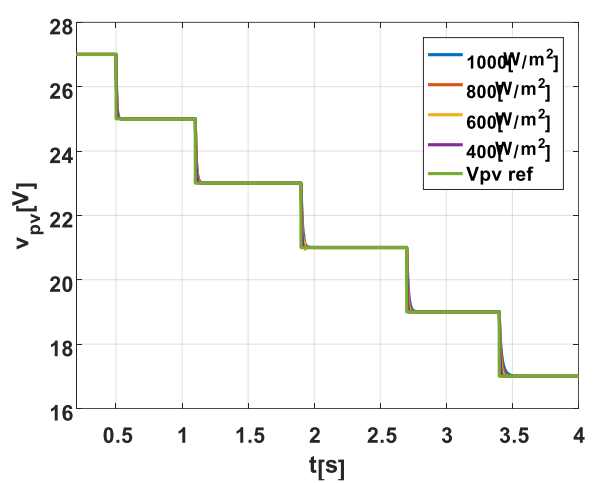

Fig. 16. Response of the adaptive controller for different irradiances.

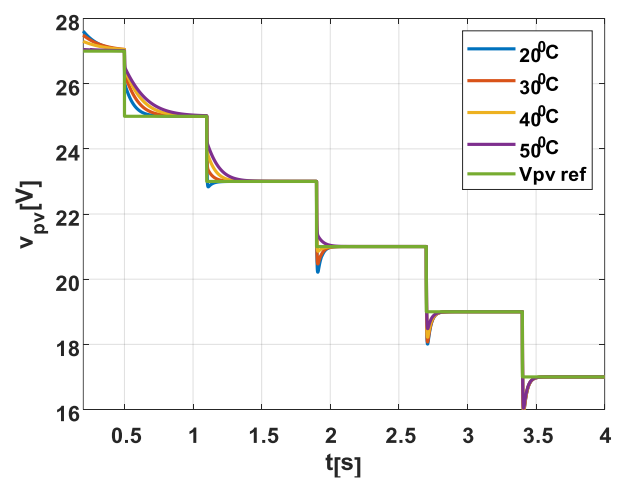

Fig. 18. Typical controller response for different temperatures. 


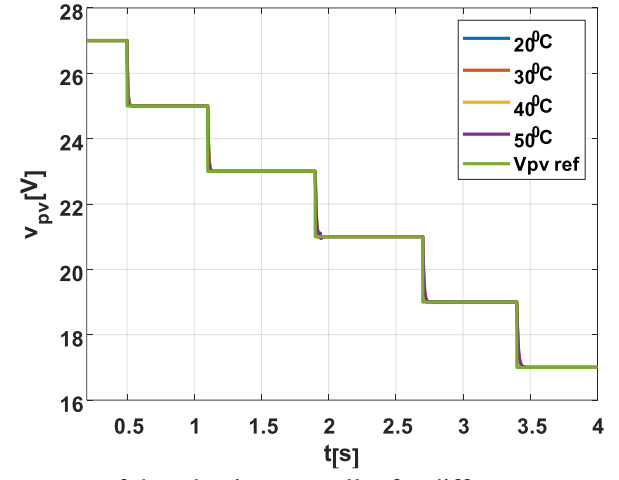

Fig. 19. Response of the adaptive controller for different temperatures.

\section{CONCLUSIONS}

This paper presented a novel control approach based on adaptive control for a PVG-BuPS-Load. The method handles the possibility of a right pole and creates an almostperfect loop gain response. Additionally, it allows you to choose the cross-over frequency without collision with the right pole. The plant is reshaped and reduced in size for simplicity and easier implantation of the adaptive control. The Bode response shows that the reduced plant has the same response as the complete plant at the frequency of interest.

In contrast to other robust methods, the adaptive control method, once implemented in PVG-BuPS-Load, allows the inverse reduced plant to counter the actual system plant at any given moment, regardless of the environmental condition and operating point. That is, a perfect loop gain response can be achieved. The method uses online dynamic conductance estimation to evaluate dynamic resistance in real-time according to the operating point and environmental conditions and injects its value into the controller. Additionally, to cope with the possibility of an unstable pole at a lower operating point, a small constant, which depends on the minimum operating point and duty cycle values, eliminates the unstable pole and has a minor influence on the system response.

The typical controller test results show a significantly uneven response, which varies from overdamped to underdamped due to the environmentally variable dependency and the nonlinear behavior of the operating point. On the other hand, test results of implanting adaptive control as a counteraction to the system plant variability clearly show that the response is stable and an almost perfect loop gain response is achieved.

\section{CONFLICTS OF INTEREST}

The authors declare that they have no conflicts of interest.

\section{REFERENCES}

[1] J. Viinamäki, J. Jokipii, T. Messo, T. Suntio, M. Sitbon, and A. Kuperman, "Comprehensive dynamic analysis of photovoltaic generator interfacing DC-DC boost power stage", IET Renewable Power Generation, vol. 9, pp. 306-314, 2015. DOI: 10.1049/IETRPG.2014.0149.

[2] M. El Fadel, G. Rachid, R. El-Samra, G. Bou Boutros, and J. Hashisho, "Knowledge management mapping and gap analysis in renewable energy: Towards a sustainable framework in developing countries", Renewable and Sustainable Energy Reviews, vol. 20, pp. 576-584, 2013. DOI: 10.1016/j.rser.2012.11.071.

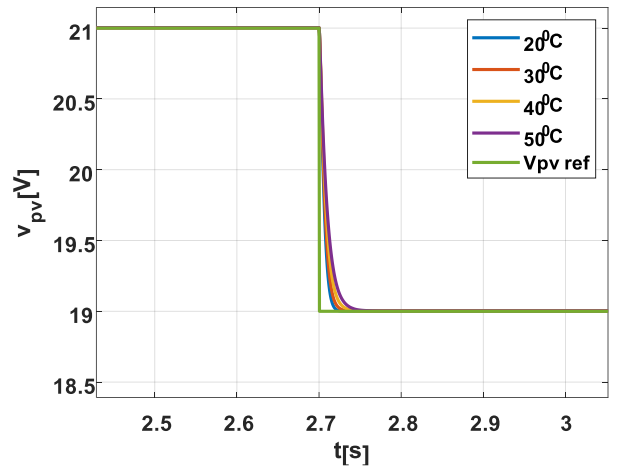

Fig. 20. Zoom-in for step response of an adaptive controller for different temperatures $\left(20^{\circ} \mathrm{C}-50{ }^{\circ} \mathrm{C}\right)$.

[3] E. H. Dursun and A. A. Kulaksiz, "Second-order fast terminal sliding mode control for MPPT of PMSG-based wind energy conversion system", Elektronika ir Elektrotechnika, vol. 26, no. 4, pp. 39-45, 2020. DOI: 10.5755/j01.eie.26.4.25762.

[4] M. Sitbon, S. Schacham, T. Suntio, and A. Kuperman, "Improved adaptive input voltage control of a solar array interfacing current mode controlled boost power stage", Energy Conversion and Management, vol. 98, pp. 369-375, 2015. DOI: 10.1016/j.enconman.2015.03.100.

[5] H. R. Baghaee, M. Mirsalim, G. B. Gharehpetian, and H. A. Talebi, "Application of RBF neural networks and unscented transformation in probabilistic power-flow of microgrids including correlated wind/PV units and plug-in hybrid electric vehicles", Simulation Modelling Practice and Theory, vol. 72, pp. 51-68, 2017. DOI 10.1016/j.simpat.2016.12.006.

[6] J. Kivimäki, S. Kolesnik, M. Sitbon, T. Suntio, and A. Kuperman, "Design guidelines for multiloop perturbative maximum power point tracking algorithms", Transactions on Power Electronics, vol. 33, no. 2, pp. 1284-1293, 2018. DOI: 10.1109/TPEL.2017.2683268.

[7] M. Muthuramalingam and P. S. Manoharan, "Comparative analysis of distributed MPPT controllers for partially shaded stand alone photovoltaic systems", Energy Conversion and Management, vol. 86, pp. 286-299, 2014. DOI: 10.1016/j.enconman.2014.05.044.

[8] M. Sitbon, M. Mellincovsky, and I. Aharon, "Controller performance assessment of a photovoltaic generator terminated in a current-modebuck-convertor-load", Elektronika ir Elektrotechnika, vol. 25, no. 3, pp. 56-62, 2019. DOI: 10.5755/j01.eie.25.3.23677.

[9] J. Zakis, E. Makovenko, H. Zeng, O. Husev, and L. Kutt, "qZS inverter as synchronverter in small-scale micro-grid", Elektronika ir Elektrotechnika, vol. 24, no. 2, pp. 58-62, Apr. 2018. DOI: 10.5755/j01.eie.24.2.20636.

[10] S. Kolesnik, M. Sitbon, S. Gadelovits, T. Suntio, and A. Kuperman, "Interfacing renewable energy sources for maximum power transfer Part II: Dynamics", Renewable and Sustainable Energy Reviews, vol. 51, pp. 1771-1783, 2015. DOI: 10.1016/j.rser.2015.04.043.

[11] M. Sitbon, I. Aharon, and M. Mellincovsky, "Robust maximum power point tracking of photovoltaic generators based on real-time dynamic conductance estimation", Energy Conversion and Management, vol 200, art. 112068, 2019. DOI: 10.1016/j.enconman.2019.112068.

[12] T. Demirdelen, R. İ. Kayaalp, and M. Tumay, "Simulation modelling and analysis of modular cascaded multilevel converter based shunt hybrid active power filter for large scale photovoltaic system interconnection", Simulation Modelling Practice and Theory, vol. 71, pp. 27-44, 2017. DOI: 10.1016/j.simpat.2016.11.003.

[13] C. M. A. da Luz, F. L. Tofoli, P. dos Santos Vicente, and E. M. Vicente, "Assessment of the ideality factor on the performance of photovoltaic modules", Energy Conversion and Management, vol. 167, pp. 63-69, 2018. DOI: 10.1016/J.ENCONMAN.2018.04.084.

[14] M. Sitbon, S. Lineykin, S. Schacham, T. Suntio, and A. Kuperman, "Online dynamic conductance estimation based maximum power point tracking of photovoltaic generators", Energy Conversion and Management, vol. 166, pp. 687-696, 2018. DOI: 10.1016/j.enconman.2018.04.053.

[15] G. Roa-Escalante, J. Sánchez-Lozano, J. Faxas, M. S. GarcíaCascales, and A. Urbina, "The effects of photovoltaic electricity injection into microgrids: Combination of geographical information systems, multicriteria decision methods and electronic control modelling", Energy Conversion and Management, vol. 96, pp. 89-99, 2015. DOI: 10.1016/J.ENCONMAN.2015.02.060.

[16] S. Baldi, A. Karagevrekis, I. T. Michailidis, and E. B. Kosmatopoulos, "Joint energy demand and thermal comfort optimization in 
photovoltaic-equipped interconnected microgrids", Energy Conversion and Management, vol. 101, pp. 352-363, 2015. DOI: 10.1016/j.enconman.2015.05.049.

[17] G. K. Singh, "Solar power generation by PV (photovoltaic) technology: A review", Energy, vol. 53, pp. 1-13, 2013. DOI: 10.1016/j.energy.2013.02.057.

[18] M. Sitbon, J. Leppäaho, T. Suntio, and A. Kuperman, "Dynamics of photovoltaic-generatorinterfacing voltage-controlled buck power stage", IEEE Journal of Photovoltaics, vol. 5, no. 2, pp. 633-640, 2015. DOI: 10.1109/JPHOTOV.2014.2379094.

[19] T. S. Mahmoud, B. S. Ahmed, and M. Y. Hassan, "The role of intelligent generation control algorithms in optimizing battery energy storage systems size in microgrids: A case study from Western Australia”, Energy Conversion and Management, vol. 196, pp. 13351352, 2019. DOI: 10.1016/j.enconman.2019.06.045.

[20] S. Gadelovits, A. Kuperman, M. Sitbon, I. Aharon, and S. Singer, "Interfacing renewable energy sources for maximum power transferPart I: Statics", Renewable and Sustainable Energy Reviews, vol. 31, pp. 501-508, 2014. DOI: 10.1016/j.rser.2013.12.039.

[21] R. Conceição, I. Vázquez, L. Fialho, and D. García, "Soiling and rainfall effect on PV technology in rural Southern Europe", Renewable Energy, vol. 156, pp. 743-747, 2020. DOI: 10.1016/j.renene.2020.04.119.

[22] L. Nousiainen et al., "Photovoltaic generator as an input source for power electronic converters", IEEE Transactions on Power Electronics, vol. 28, no. 6, pp. 3028-3038, 2013. DOI: 10.1109/TPEL.2012.2209899.

[23] M. Sitbon, S. Schacham, and A. Kuperman, "Disturbance observer based voltage regulation of current-mode-boost-converter-interfaced photovoltaic generator", IEEE Transactions on Industrial Electronics, vol. 62, no. 9, pp. 5776-5785, 2015. DOI: 10.1109/TIE.2015.2434796.

[24] M. Sitbon, I. Aharon, M. Averbukh, D. Baimel, and M. Sassonker (Elkayam), "Disturbance observer based robust voltage control of photovoltaic generator interfaced by current mode buck converter", Energy Conversion and Management, vol. 209, art. 112622, 2020. DOI: 10.1016/j.enconman.2020.112622.

[25] Z. Zhang, X. Cheng, and J. Liu, "An improvement method for extracting five parameters of a solar cell based on Lambert Wfunction with the current-voltage data", Applied Mechanics and Materials, vols. 291-294, pp. 38-42, 2013. DOI: 10.4028/www.scientific.net/AMM.291-294.38.

[26] F. Ghani and M. Duke, "Numerical determination of parasitic resistances of a solar cell using the Lambert W-function", Solar Energy, vol. 85, pp. 2386-2394, 2011. DOI: 10.1016/J.SOLENER.2011.07.001.

[27] M. Villalva, T. G. de Siqueira, and E. Ruppert, "Voltage regulation of photovoltaic arrays: Small-signal analysis and control design", IET Power Electronics, vol. 3, no. 6, pp. 869-880, 2010. DOI: 10.1049/iet-pel.2008.0344.

[28] S. Gadelovits, M. Sitbon, T. Suntio, and A. Kuperman, "Single-source multibattery solar charger: Case study and implementation issues", Progress in Photovoltaics: Research and Applications, vol. 23, pp. 1916-1928, 2015. DOI: 10.1002/pip.2591

[29] G. Petrone, G. Spagnuolo, R. Teodoresku, M. Veerachary, and M. Vitelli, "Reliability issues in photovoltaic power processing systems", IEEE Transactions on Industrial Electronics, vol. 55, no. 7, pp. 25692580, 2008. DOI: 10.1109/TIE.2008.924016.

This article is an open access article distributed under the terms and conditions of the Creative Commons Attribution 4.0 (CC BY 4.0) license (http://creativecommons.org/licenses/by/4.0/) 